\title{
Intracranial Mesenchymal Chondrosarcoma: A Case Report
}

\author{
Prachi Agrawal $^{1}$ Ajay Gupta ${ }^{1}$ Shagun Juneja ${ }^{1}$ Veena Gupta ${ }^{1}$ \\ ${ }^{1}$ Department of Radiotherapy, Safdarjung Hospital, New Delhi, India \\ Address for correspondence Dr. Prachi Agrawal, DNB, C1/47, Second \\ Floor, Janakpuri, New Delhi 110058, India (e-mail: dr_prachi@ymail.com).
} Indian J Neurosurg 2015;4:31-34.

\begin{abstract}
Primary intracranial mesenchymal chondrosarcoma is an extremely rare tumor. We present a case of primary intracranial mesenchymal chondrosarcoma in a young female who presented with headache, loss of vision, and one episode of convulsions associated with nausea and vomiting. MRI brain revealed a large, well-marginated, lobulated, partially calcified, intensely enhancing lesion with a broad dural base in bilateral frontal regions with marked mass effect suggestive of meningioma. Bilateral frontal craniotomy with gross tumor excision was performed. Histopathology revealed

Keywords

- mesenchymal chondrosarcoma

- intracranial

- surgery

- radiotherapy mesenchymal chondrosarcoma. Adjuvant radiotherapy was delivered and patient is currently on follow-up. We emphasize that intracranial mesenchymal chondrosarcoma is a rare neoplasm and should be considered in the differential diagnoses of intracranial mass along with meningioma. Treatment of choice is surgery followed by adjuvant radiotherapy. We review the relevant literature and discuss the characteristics of this rare tumor.
\end{abstract}

\section{Introduction}

Mesenchymal chondrosarcoma is a rare tumor, first described by Lightenstein and Bernstein in 1959. ${ }^{1}$ It develops in bones, especially the pelvis, ribs, femur, humerus, vertebrae, and mandible and is less commonly found in somatic soft tissue. ${ }^{1,2}$ However, an extraosseous origin has been reported in almost one-third of patients, and of these the brain is the most frequent site. ${ }^{3,4}$

The case of an 18-year-old woman is discussed, who presented with primary intracranial mesenchymal chondrosarcoma that originated from dura in bilateral frontal region. We present the clinical, radiological, and histopathological features of these tumors with their treatment and review the relevant literature.

\section{Case Report}

The patient presented with a 6-month history of dull headache in frontal region and progressive loss of vision in both eyes for the last 4 months. At presentation, she gave history of one episode of convulsions about 15 days back associated with nausea and vomiting. The only abnormality on neurological examination was the loss of vision in both eyes. On ocular examination, perception of light was absent in both eyes. There was loss of pupillary reflex in the right eye, while it was present in the left eye. Proptosis was observed but nystagmus was not seen. Glabellar tap was present. On fundus examination, bilateral discs were pale with secondary optic atrophy.

Contrast-enhanced cranial CT scan showed ill-defined hypodense to hyperdense lesion in bilateral frontal region with hyperostosis of bone suggestive of frontal meningioma.

MRI scan of brain revealed a well-marginated, lobulated, extra-axial lesion $\sim 9.3 \times 6.5 \times 6 \mathrm{~cm}$ in maximum dimensions, with a broad dural base toward the calvarium. The lesion was seen in bilateral frontal region crossing the midline. It caused buckling of underlying gray-white matter with a cerebrospinal fluid cleft separating the mass from the adjacent cerebral parenchyma. There was marked perifocal edema, which appeared to be abutting the anterior aspect of the superior sagittal sinus. The lesion had isointense to hypointense signal on $\mathrm{T} 1$-weighted images and hyperintense signal on T2-weighted and fluid-attenuated inversion recovery images, with interspersed hypointense foci showing "blooming effect" on GRE images suggesting a published online January 21, 2015
DOI http://dx.doi.org/ 10.1055/s-0035-1548997. ISSN 2277-954X. (c) 2015 Neurological Surgeons' Society of India

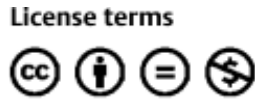




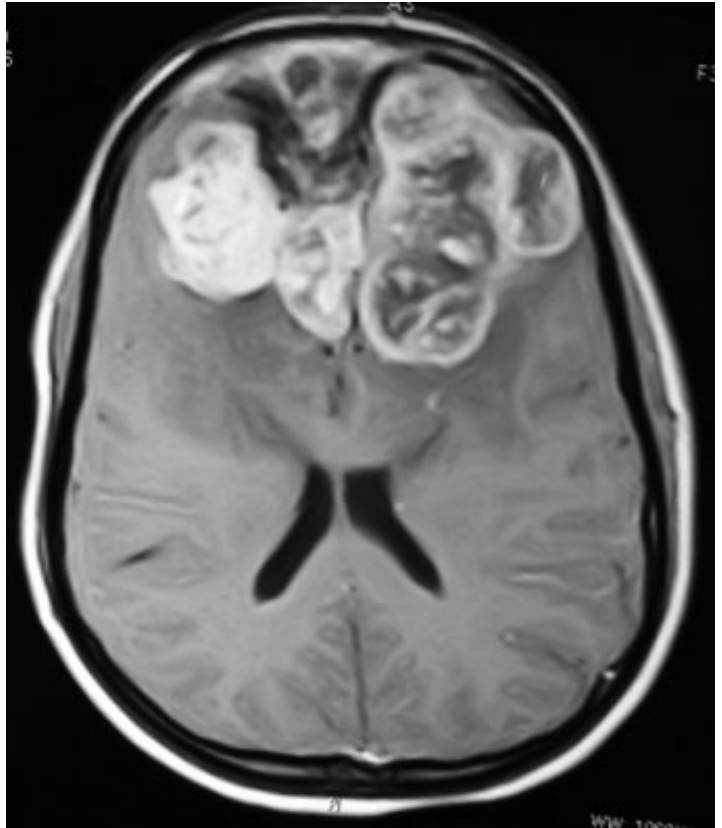

Fig. 1 T1-weighted MRI with gadolinium showing a large lobular, heterogeneously enhancing mass in bilateral frontal region.

partially calcified lesion (-Figs. $\mathbf{1}$ and $\mathbf{2}$ ). It caused marked mass effect in the form of compression of the surrounding brain parenchyma, compression and displacement of the corpus callosum posteroinferiorly, and compression of the frontal horns of bilateral lateral ventricles. It also appeared to cause mild mass effect on optic chiasma. Findings were consistent with possibility of a partially calcified meningioma.

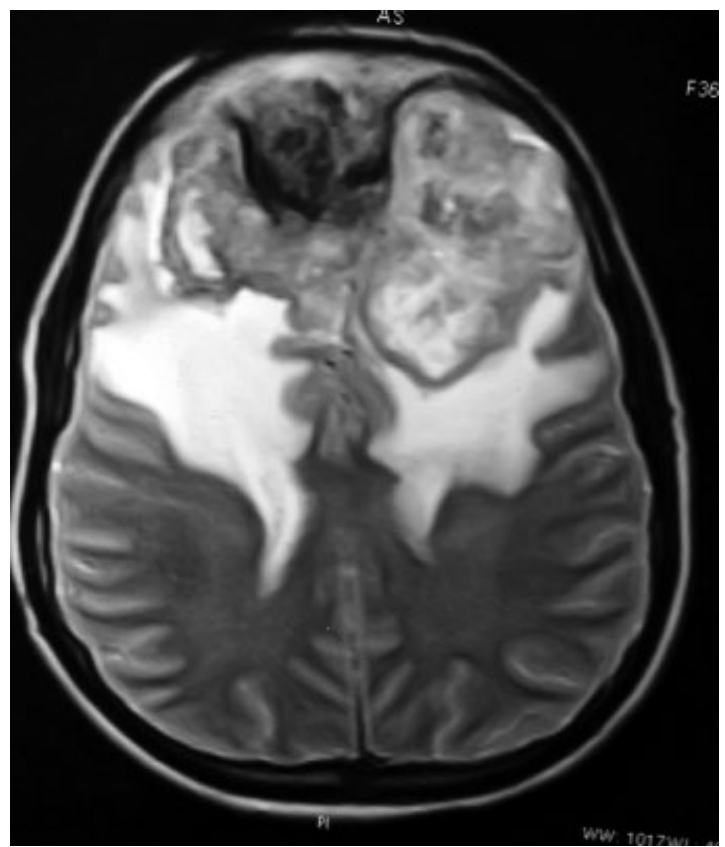

Fig. 2 T2-weighted MRI showing cystic components and surrounding edema.
Bilateral frontal craniotomy was performed and gross tumor excision was achieved. During surgery, part of tumor was firmly adhered to the bone and came out along with bone flap. Surface of dura was eroded on both sides. Superior sagittal sinus was invaded by the tumor. Total bone flap, dura along with falx cerebri, and whole of the tumor were removed.

\section{Pathological Findings}

Macroscopically, the tumor was pale with gray and glistening surface. It had a nodular and lobulated chondroid appearance with scattered yellow calcification.

Microscopically, a cellular tumor composed of small round to polygonal cells which were arranged in sheets and trabecular pattern was found. In the surrounding area, cartilaginous tissue could be identified with well-formed cartilages at some places. In both the components, the mitotic rate was extremely low. On immunohistochemistry, tumor stained positive for vimentin in cartilaginous area and for CD-99 in small cell area but was negative for S-100. Features were consistent with intracranial mesenchymal chondrosarcoma.

The patient received adjuvant radiotherapy 6000 cGy in 30 fractions by conventional fractionation over 6 weeks on Tele-Cobalt unit (Theratron 780E). Initially, a wider field was planned with $5-\mathrm{cm}$ margin, which was coned down to $2-\mathrm{cm}$ margin after $46 \mathrm{~Gy} / 23$ fractions. She remains in a good medical condition and is on regular follow-up.

\section{Discussion}

Primary intracranial cartilaginous tumors, which include chondroma, chondrosarcoma, etc., are rare. Chondrosarcoma accounts for $6 \%$ of skull base neoplasms and $0.15 \%$ of all intracranial tumors. ${ }^{5}$

Chondrosarcomas are malignant cartilaginous neoplasms that originate from bone, cartilage, and mesenchymal soft tissue. They are classified as skeletal or mesenchymal types. Both types of chondrosarcoma, each with a distinct morphological appearance, can arise from bone and extraosseous mesenchymal tissue. Pure skeletal type extraosseous intracranial chondrosarcoma is rare and seems to have a better prognosis. ${ }^{6}$

In 1959, Lightenstein and Bernstein were the first to differentiate mesenchymal chondrosarcoma from other cartilaginous tumors histologically. ${ }^{1}$ The pluripotent mesenchymal cell, from which all types of connective tissue is derived, is hypothesized as the cell of origin. ${ }^{7}$ Mesenchymal chondrosarcoma generally develops in bones, especially the pelvis, ribs, femur, humerus, vertebrae, and mandible., ${ }^{1,2}$ Extraosseous origin can be divided into two subgroups: (1) associated with soft tissue and muscle and (2) associated with central nervous system. ${ }^{3}$ Brain is the most commonly reported extraosseous site of origin. ${ }^{2,4,7}$

One postulated theory for the development of intracranial chondrosarcoma highlights that while the bones of the skull vault develop primarily by 
intramembranous ossification, the bones of the skull base mature predominantly by endochondral ossification. ${ }^{8}$ Endochondral ossification is also responsible for the development of several sites in the mature skull including a large part of the petrous portion of the temporal bone, areas of the petro-occipital, spheno-occipital, and sphenopetrosal synchondrosis. Intracranial chondrosarcomas might develop from the chondrocytes within rests of endochondral cartilage. Others believe that the primitive multifunctional mesenchymal cells involved in the embryogenesis of the skull base and temporal bone may be the source of these tumors. Yet, other authors contend that intracranial chondrosarcomas develop from metaplastic mature fibroblasts. ${ }^{9,10}$

Although most chondrosarcomas arise de novo, they are common in patients with Ollier disease, Maffucci syndrome, Paget disease, and osteochondroma. ${ }^{5}$

They are subclassified into the conventional (hyaline/ myxoid), dedifferentiated, clear cell, and mesenchymal subtypes. ${ }^{5}$ The conventional subtype of chondrosarcoma is the most common type of cartilage forming tumor to develop in skull base. ${ }^{11}$ It is composed of either hyaline cartilage or myxoid cartilage, or a combination of both of these matrices. The hyaline subtype is characterized by hypercellular hyaline cartilage containing cytologically atypical chondrocytes within lacunae, while the atypical chondrocytes of the myxoid subtype do not reside in lacunae but instead are enmeshed in a flocculent myxoid matrix. Intracranial occurrence of clear cell or dedifferentiated subtypes is very rare.

The mesenchymal subtype displays a more anaplastic appearance with cellular mixture of small spindle-shaped anaplastic cells, frequently located around small vessels in chondroid tissue at various stages of development. They have a characteristic biomorphic pattern, and are made up of islands of hyaline cartilage within a population of small, undifferentiated, mesangial cells. Although the histopathological appearance of this tumor is quite distinct, their rarity, their frequent occurrence in the meninges, and their highly vascular structure can lead to the erroneous diagnosis of angioblastic meningioma or hemangiopericytoma.

Rushing et al reported immunoreactivity of the S-100 protein in the cartilaginous islands and immunoreactivity of vimentin in the undifferentiated component. ${ }^{12}$ Because of the rarity of this tumor, immunohistochemical analyses can help to confirm the diagnosis and differentiate this type of tumor from the hemangiopericytoma and cartilaginous meningioma.

Although there is significance of histological subtypes of chondrosarcoma, the prognosis, however, is determined primarily by World Health Organization (WHO) histological grade. The grading system consists of three categories: grade 1 (well differentiated), grade 2 (moderately differentiated), and grade 3 (poorly differentiated).

There are studies reporting occurrence of intracranial mesenchymal chondrosarcoma from 2 months to 71 years of age; it occurs most frequently between 20 and 30 years, with slight female predominance. $^{13}$ Our patient was 18 years old.

These tumors are well formed and usually large at the time of diagnosis. They show progressive enlargement and manifest clinically by producing pressure symptoms or invasion of local structures such as brain, cranial nerves, and optic pathways, hence causing various symptoms such as diplopia, headache, and convulsions. Our patient had complaints of headache and progressive vision loss in bilateral eyes. She also had one episode of convulsions.

The radiological features of chondrosarcoma are the absence of a dural tail, minimal vasogenic edema, and relative avascularity on angiography. On MRI, it shows higher signal intensity on T2-weighted images and islands of low-signal intensity despite a lack of gross calcification on all sequences, which is due to well-differentiated cartilage. They are often diagnosed as meningioma or hemangiopericytoma on radiological examination. ${ }^{2,4,7}$

The complete surgical resection is the mainstay of treatment, although it is difficult to achieve, as these tumors are located deeply. Because of high malignant potential, high incidence of local recurrence, and incomplete resection, surgery followed by adjuvant radiotherapy has become the standard treatment of intracranial mesenchymal chondrosarcoma. ${ }^{14-17}$ Radiotherapy has been shown to increase the tumor-free recurrence interval. It has been delivered in the literature using conventional external beam radiation therapy, radiosurgery, proton beam radiation, CyberKnife, and carbon ion.

Although adjuvant radiation therapy appears to reduce the mortality rate of chondrosarcomas, further investigations into the usefulness of adjuvant therapy for completely resected, low-grade chondrosarcomas must be performed. It is possible that low-grade chondrosarcomas have good survival with surgery alone and are better treated with resection without immediate radiotherapy or with delayed adjuvant radiation.

Postoperative radiation therapy has been stated to be beneficial in these patients by Neff et al in 2002 and Isaacson et al in 2007. ${ }^{18,19}$ Bloch et al in 2009 showed that patients treated with surgery alone for intracranial chondrosarcoma demonstrated a 5 -year mortality rate of $26 \%$, while the implementation of postoperative adjuvant radiation therapy reduced this mortality rate dramatically to $4 \%{ }^{5}$

The role of chemotherapy is not established in chondrosarcoma. There are studies using temozolomide and ifosfamide-doxorubicin combination chemotherapy in intracranial mesenchymal chondrosarcoma successfully. ${ }^{20,21}$

Because of rarity of these tumors and hence lack of proper literature, there is significant interpractitioner variability in management of these tumors, as most of the studies are case studies or small- to modest-sized case series without significant statistical analysis. Because of its rarity and therefore limited experience, it is difficult to draw conclusions about the appropriate adjuvant treatment.

The prognosis of patients with intracranial chondrosarcoma is strongly influenced by several significant 
factors such as the extent of surgical excision, the use of adjuvant radiation therapy, and pathological pattern. However, local recurrence is considered by many to be the most significant predictor of mortality in these patients.

The mesenchymal subtype indicates worse clinical behavior and poorer prognosis than conventional histology according to Oikawa et al. ${ }^{22}$ In a study conducted by Bloch et al, 5-year mortality rate for conventional subtype was $6 \%$, while for mesenchymal subtype it was $54 \%^{5}$

Bloch et al in 2009 performed a systematic analysis of survival in a large population of patients of intracranial chondrosarcomas. The overall 5-year mortality among patients in their study was $11 \%$, with an average survival time of 53.7 months (median, 24 months), which was comparable to those quoted in the literature. ${ }^{5}$

\section{Conclusion}

Intracranial mesenchymal chondrosarcoma is an extremely rare neoplasm which should be considered in differential diagnoses of intracranial mass presenting clinically and radiologically as meningioma, especially in young adults. Conventional variant has better prognosis than mesenchymal type. Radical surgical excision with wide margins, followed by adjuvant radiotherapy, is recommended as the treatment of choice.

\section{Conflict of Interest}

Nil.

\section{Source of Support}

Nil.

\section{Presentation at a Meeting}

None.

\section{References}

1 Lightenstein L, Bernstein D. Unusual benign and malignant chondroid tumors of bone. A survey of some mesenchymal cartilage tumors and malignant chondroblastic tumors, including a few multicentric ones, as well as many atypical benign chondroblastomas and chondromyxoid fibromas. Cancer 1959;12: 1142-1157

2 Nakashima Y, Unni KK, Shives TC, Swee RG, Dahlin DC. Mesenchymal chondrosarcoma of bone and soft tissue. A review of 111 cases. Cancer 1986;57(12):2444-2453

3 Hassounah M, Al-Mefty O, Akhtar M, Jinkins JR, Fox JL. Primary cranial and intracranial chondrosarcoma. A survey. Acta Neurochir (Wien) 1985;78(3-4):123-132
4 Scheithauer BW, Rubinstein LJ. Meningeal mesenchymal chondrosarcoma: report of 8 cases with review of the literature. Cancer 1978;42(6):2744-2752

5 Bloch OG, Jian BJ, Yang I, et al. A systematic review of intracranial chondrosarcoma and survival. J Clin Neurosci 2009;16(12): 1547-1551

6 Alvira MM, McLaurin RL. Asymptomatic subdural chondrosarcoma. Case report. J Neurosurg 1978;48(5):825-828

7 Harsh GR IV, Wilson CB. Central nervous system mesenchymal chondrosarcoma. Case report. J Neurosurg 1984;61(2):375-381

8 Lau DP, Wharton SB, Antoun NM, Bottrill ID, Moffat DA. Chondrosarcoma of the petrous apex. Dilemmas in diagnosis and treatment. J Laryngol Otol 1997;111(4):368-371

9 Coltrera MD, Googe PB, Harrist TJ, Hyams VJ, Schiller AL, Goodman ML. Chondrosarcoma of the temporal bone. Diagnosis and treatment of 13 cases and review of the literature. Cancer 1986; 58(12):2689-2696

10 Seidman MD, Nichols RD, Raju UB, Mehta B, Levy HG. Extracranial skull base chondrosarcoma. Ear Nose Throat J 1989;68(8): 626-632, 635

11 Korten AG, ter Berg HJ, Spincemaille GH, van der Laan RT, Van de Wel AM. Intracranial chondrosarcoma: review of the literature and report of 15 cases. J Neurol Neurosurg Psychiatry 1998;65(1): 88-92

12 Rushing EJ, Armonda RA, Ansari Q Mena H. Mesenchymal chondrosarcoma: a clinicopathologic and flow cytometric study of 13 cases presenting in the central nervous system. Cancer 1996; 77(9):1884-1891

13 Vergeer RA, Vink R, Avenarius JK, Driesse MJ. A 71-year-old woman with an intracranial dural-based mesenchymal chondrosarcoma. J Clin Neurosci 2012;19(8):1170-1171

14 Austin-Seymour M, Munzenrider J, Goitein M, et al. Fractionated proton radiation therapy of chordoma and low-grade chondrosarcoma of the base of the skull. J Neurosurg 1989; 70(1):13-17

15 Gay E, Sekhar LN, Rubinstein E, et al. Chordomas and chondrosarcomas of the cranial base: results and follow-up of 60 patients. Neurosurgery 1995;36(5):887-896, discussion 896-897

16 Sen CN, Sekhar LN, Schramm VL, Janecka IP. Chordoma and chondrosarcoma of the cranial base: an 8-year experience. Neurosurgery 1989;25(6):931-940, discussion 940-941

17 Stapleton SR, Wilkins PR, Archer DJ, Uttley D. Chondrosarcoma of the skull base: a series of eight cases. Neurosurgery 1993;32(3): 348-355, discussion 355-356

18 Neff B, Sataloff RT, Storey L, Hawkshaw M, Spiegel JR. Chondrosarcoma of the skull base. Laryngoscope 2002;112(1): 134-139

19 Isaacson B, Kutz JW, Roland PS. Lesions of the petrous apex: diagnosis and management. Otolaryngol Clin North Am 2007; 40(3):479-519, viii

20 Aksoy S, Abali H, Kiliçkap S, Güler N. Successful treatment of a chemoresistant tumor with temozolomide in an adult patient: report of a recurrent intracranial mesenchymal chondrosarcoma. J Neurooncol 2005;71(3):333-334

21 Sardi I, Massimino M, Genitori L, Buccoliero AM, Giangaspero F, Ferrari A. Intracranial mesenchymal chondrosarcoma: report of two pediatric cases. Pediatr Blood Cancer 2011;56(4):685-686

22 Oikawa H, Satoh T, Masuda T, Arai H, Ehara S, Muro-Oka G. Intracranial low-grade chondrosarcoma with hyperostosis of the skull: a case report. J Neurooncol 2000;49(3):249-254 